\title{
Novel ETHE1 mutation in a carrier couple having prior offspring affected with ethylmalonic encephalopathy: Genetic analysis, clinical management and reproductive outcome
}

\author{
DAVID J. WALSH ${ }^{1}$, ERIC SCOTT SILLS ${ }^{1}$, DEBORAH M. LAMBERT ${ }^{2}$, \\ NILS GREGERSEN ${ }^{4}$, FERGAL D. MALONE ${ }^{3}$ and ANTHONY P.H. WALSH ${ }^{1}$

\begin{abstract}
${ }^{1}$ The Sims Institute/Sims International Fertility Clinic; ${ }^{2}$ Department of Genetics, Children's University Hospital;
\end{abstract} \\ ${ }^{3}$ Department of Obstetrics and Gynaecology, School of Medicine, Royal College of Surgeons in Ireland, Dublin, Ireland; \\ ${ }^{4}$ Research Unit for Molecular Medicine, Aarhus University Hospital, Skejby, Denmark
}

Received October 21, 2009; Accepted November 27, 2009

DOI: $10.3892 / \mathrm{mmr} 00000243$

\begin{abstract}
Ethylmalonic encephalopathy (EE) is an autosomally recessive inherited disorder with a relentlessly progressive decline in neurological function, usually fatal by the age of ten. It is characterised by generalised hypotonia, psychomotor regression, spastic tetraparesis, dystonia, seizures and, eventually, global neurological failure. Approximately 50 reports have been published worldwide describing this devastating disease, most involving patients of Mediterranean or Arab origin. The fundamental defect in EE likely involves the impairment of a mitochondrial sulphur dioxygenase coded by the ETHE1 gene responsible for the catabolism of sulphide, which subsequently accumulates to toxic levels. A diagnosis of EE should initiate careful genetic evaluation and counselling, particularly if the parents intend to have additional offspring. The present report describes the diagnosis of EE in a reproductive endocrinology context, where both members of a non-consanguineous couple were confirmed to be carriers of an identical $A \rightarrow G$ mutation. This previously unknown mutation at nucleotide position c.494 resulted in an amino acid substitution, p.Asp165Gly. Although consideration was given to in vitro fertilisation, embryo biopsy and single gene pre-implantation genetic diagnosis, the couple decided to first utilise a less aggressive therapeutic approach with donor sperm insemination. Pregnancy with a low risk of EE was indeed achieved; however, the infant was affected with a different anomaly (hypoplastic left heart). As this case demonstrates, prior to the initiation of fertility therapy, genetic analysis may be used to provide a confirmatory diagnosis when $\mathrm{EE}$ is suspected.
\end{abstract}

Correspondence to: Dr Eric Scott Sills, The Sims Institute/Sims International Fertility Clinic, Rosemount Hall, Dundrum Road, Dundrum, Dublin 14, Ireland

E-mail:drscottsills@sims.ie

Key words: ethylmalonic encephalopathy, inherited disorder, hypoplastic left heart defect

\section{Case report}

Clinical presentation. The patient, aged 361/2 years, sought reproductive endocrinology consultation with her husband. She suffered from mild hypothyroidism and pernicious anaemia, which were medically managed and stable before presentation. Her blood type was $\mathrm{O}^{+}$. Neither partner smoked and both were in good general health. The ethnicity of both partners was Caucasian, and there was no family history of any genetic condition. Consanguinity was absent. The couple had achieved a total of four pregnancies together, none of which had required medical assistance. The first two pregnancies yielded term male deliveries, both via caesarean. The second two pregnancies ended in miscarriage, one each in the first and second trimester. The second delivery resulted in the birth of a son who evidenced developmental delay at the age of approximately six months. Paediatric neurology assessment of this infant included EEG and brain MR, which revealed non-specific abnormalities. Initial investigations for spastic diplegia and global developmental delay included the measurement of blood lactate, which was elevated, and of urine organic acids, which revealed high ethylmalonic acid and methylsuccinate. A defect in fatty acid oxidation was thus suspected, with short chain acyl-coA dehydrogenase deficiency (SCAD) and ethylmalonic aciduria considered as provisional diagnoses. A study of fatty acid $\beta$-oxidation in fibroblasts excluded medium- and long-chain fatty acid defects. The serum acylcarnitine profile revealed elevated butyrylcarnitine, which did not exclude SCAD. Muscle biopsy showed diminished cytochrome oxidase; spectrophotometric dosage of respiratory chain complexes on the sample confirmed deficiency of mitochondrial complex IV. Gene sequencing for SCAD and ethylmalonic encephalopathy identified no mutations in the ACADS gene (SCAD), but did identify a novel mutation in ETHE1. This mutation was determined to be homozygous by confirmation of carrier status via blood samples from both parents of the proband. A treatment regimen was started for the proband, and the parents were advised that any future pregnancies would have a $25 \%$ risk of being affected with EE. The parents understood that the metabolic derangements associated with the mitochondrial 


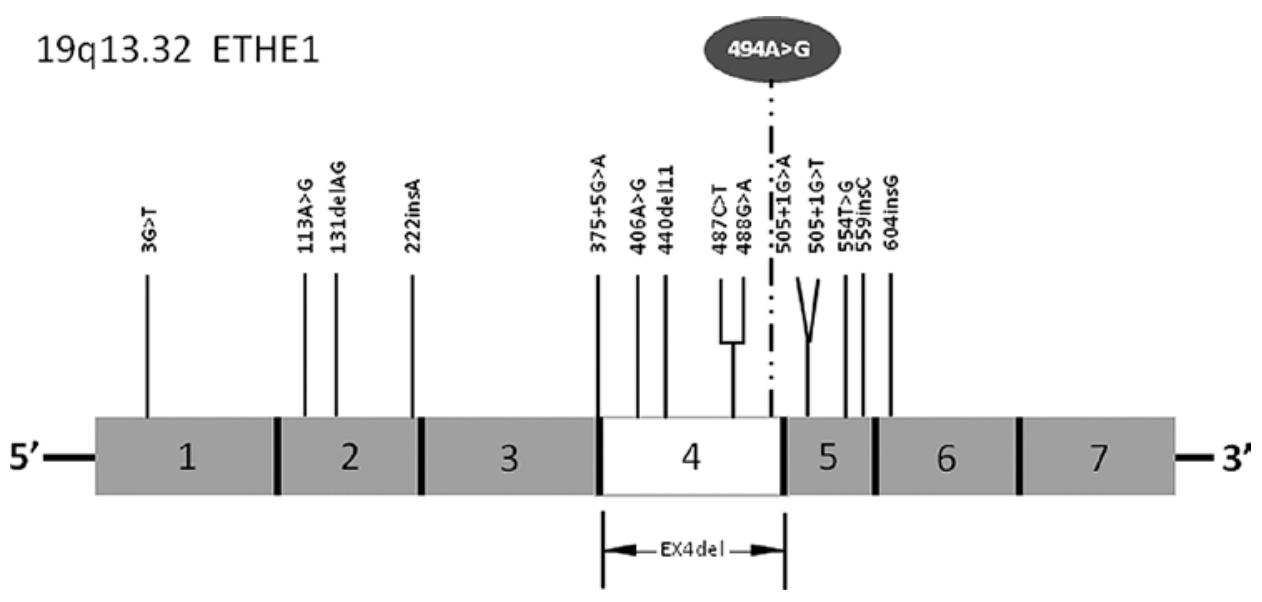

Figure 1. Diagram of the ETHE1 gene (19q13.32) with previously reported representative mutations and associated exon for each. A new $A \rightarrow G$ mutation at c.494 (circled above exon4) was associated with a p.Asp165Gly substitution.

abnormalities were secondary to EE, and did not constitute a separate diagnosis.

The couple were seen by a genetic counsellor over a twoyear period. They were aware that early implementation of any therapy for a baby affected with EE would not change the course of disease in that child. Treatment options included gonadotropin ovulation induction and insemination (IUI) using anonymous donor sperm or, if native gametes were preferred, IVF incorporating pre-implantation genetic diagnosis (PGD), which permits the selection of unaffected embryos prior to transfer. As EE is a very rare condition with a hypothetical maximum 1:250 carrier rate under Hardy-Weinberg equilibrium, IUI using anonymous donor sperm would result in a risk of $<1: 1000$ of offspring having EE.

At our centre, a gynaecological exam identified no abnormalities, and transvaginal saline infusion ultrasound (with enhancement) confirmed an unremarkable intrauterine contour and fallopian tubal patency. Pap test and cervical cultures were negative. The patient was confirmed to have mild PCOS, and the administration of $500 \mathrm{mg}$ glucophage thrice daily, which had been started about one year previously, was maintained. Thrombophilia panel and other laboratory tests were normal, although a substantially elevated anti-thyroid antibody titre of $115.2 \mathrm{IU} / \mathrm{ml}$ (reference range $0-4.11 \mathrm{IU} / \mathrm{ml}$ ) was noted. Additionally, anti-thyroid peroxidise antibody was $>1,000 \mathrm{kIU} / 1$ (reference range 0-5.6 kIU/l) and serum IgA titre was marginally high at $2.96 \mathrm{~g} / \mathrm{l}$ (reference range 0.84-2.69). Pulsed Doppler ultrasound of the thyroid was negative for nodularity or abnormal flow patterns. The husband's semen analysis was normal.

Sequencing protocol. DNA was supplied as blood spots on Guthrie cards or purified from whole blood. Generations reagents (Gentra Systems, Minneapolis, MN, USA) were used to isolate DNA. References to nucleotides or amino acids were based on the cDNA sequence of ETHE1 (NM 014297), so that the initiating ATG codon was numbered as bp 1-3 and the initiator methionine was designated amino acid 1. Primers for PCR amplification of the seven ETHE1 exons were configured as previously described (1). The following primers were tagged with a terminal M13 nucleotide sequence to facilitate subsequent cycle sequence reactions: exon1 F: 5'-TGTAAAACGACGGCC AGTTCCGTGGCCCCTTTAGGCGT-3'; exon1 R: 5'-CAG GAAACAGCTATGACCCCCGGAGTTCCGTCCTTGCT-3'; exon2 F: 5'-TGTAAAACGACGGCCAGTAGCAAGGACGG AACTCCGGG-3'; exon2 R: 5'-CAGGAAACAGCTATGACC TCCCATTCCACTGACGCTGCA-3'; exon3 F: 5'-TGTAAA ACGACGGCCAGTTGCAGCGTCAGTGGAATGGA-3'; exon3 R: 5'-CAGGAAACAGCTATGACCAAGAAGTCCAG GCCACCACC-3'; exon4 F: 5'-TGTAAAACGACGGCCAGTG GGCCAGTTTCATCTAGAAGGC-3'; exon4 R: 5'-CAGGAA ACAGCTATGACCGCCCCCTAAAAGTCTAATGTCC-3'; exon5F:5'-TGTAAAACGACGGCCAGTGGAAGGGGTTAG AGTCTTCTGT-3'; exon5 R: 5'-CAGGAAACAGCTATGACC GAGACTGGTCGTCTTCATGCC-3'; exon6 F: 5'-TGTAAA ACGACGGCCAGTAGTTCTGAGAGGCCTGAGGCA-3'; exon6R:5'-CAGGAAACAGCTATGACCACCCAGGAGTCC AAGACCCCA-3'; exon7 F: 5'-TGTAAAACGACGGCCAGT AGTGGGGCCTGGAACTCTAC-3'; exon7 R: 5'-CAGGAA ACAGCTATGACCCGGCCCAGAAACCCAATTGG-3'.

PCR amplification was conducted at $96^{\circ} \mathrm{C}$ for $15 \mathrm{~min}$ (initial cycle) followed by 40 cycles at $95^{\circ} \mathrm{C}$ for $1 \mathrm{~min}, 59^{\circ} \mathrm{C}$ for $30 \mathrm{sec}$ and $72^{\circ} \mathrm{C}$ for $2 \mathrm{~min}$. A final cycle was carried out at $72^{\circ} \mathrm{C}$ for 5 min. Sequence reactions were prepared using the BigDye Terminator kit 1.1 (Applied Biosystems, Foster City, CA, USA). Nucleotide sequence data were collected on an ABI PRISM ${ }^{\circledR}$ 3100-Avant Genetic Analyzer and analysed via Sequencher v3.1 (Gene Codes Corp., Ann Arbor, MI, USA). Sequence primers were forward and reverse M13 oligonucleotides, except for the reverse primer to the exon6 sequence. Due to repeat sequences at intron6, a specific sequence primer was used: 5'-AGTCCA GGTCCCCAGCTG-3'.

Results and clinical outcome. Genotype analysis revealed that both parents had an identical $\mathrm{A} \rightarrow \mathrm{G}$ mutation at nucleotide position c.494, resulting in an amino acid substitution, p.Asp165Gly (Fig. 1). This specific mutation has not been previously identified (2), but is considered to be diseaseassociated as it was absent in 56 control individuals, and no other amino acid-modifying sequence alteration was detected in the analysed sequences. Follow-up consultation with a genetic counsellor was provided. Since the couple desired another 
pregnancy, they were offered either ovulation induction with intrauterine insemination (IUI) using anonymous donor sperm or (if native gametes were preferred) IVF with pre-implantation genetic diagnosis before transfer to select unaffected embryos. They decided to pursue IUI, and a 75 IU/day r-FSH regime was initiated without pituitary down-regulation. Given the anti-thyroid antibody titre and history of two miscarriages before presentation, 5,000 $\mu /$ day s.c. heparin, $20 \mathrm{mg} /$ day oral prednisolone and $75 \mathrm{mg} /$ day aspirin was started empirically at the time of follicular recruitment $(3,4)$. The ovulation induction response was monitored by periodic follicular assessment via transvaginal ultrasound and serum estradiol determinations. Donor insemination was performed $36 \mathrm{~h}$ after 10,000 IU s.c. hCG administration to trigger ovulation. Though the first IUI cycle failed to achieve pregnancy, the patient returned the following month and a second attempt using the same protocol was undertaken. For both treatment cycles, the same anonymous donor was used, with samples obtained from a Danish sperm bank. Fourteen days after her second IUI, a positive pregnancy test was obtained. Transvaginal ultrasound confirmed a single intrauterine pregnancy at a gestational age of seven weeks, with positive embryonic cardiac action (rate 107/min). Antepartum surveillance by targeted (level II) ultrasound was consistent with hypoplastic left heart syndrome, a diagnosis confirmed during additional testing performed during the second trimester. Amniocentesis revealed a euploid pregnancy with an inversion of $5 q 12 q 13$. FISH analysis showed normal coverage (whole chromosome staining) for both chromosome 5 homologues and no 22q11.2 deletion. The couple declined karyotyping to determine whether this was an inherited or de novo disorder. Sadly, an intrauterine fetal demise occurred at approximately 32 weeks, and placenta accreta necessitated a caesarean hysterectomy.

\section{Discussion}

This is the first published description of ethylmalonic encephalopathy (EE) encountered in a clinical infertility setting, and the only known case from Ireland. EE is a very rare autosomal recessive inborn error of metabolism caused by mutations in the ETHE1 gene localised to chromosome 19q13. A mutation at this site has been shown to result in functional impairment of a mitochondrial sulphur dioxygenase, responsible for sulphide catabolism (5). While the exact incidence of EE is unknown, only some 50 cases have been reported worldwide (2). Thus far, most affected individuals have been of Arab or Mediterranean origin.

EE usually manifests as neurological delay within the first year of life, and is progressively catastrophic. It is typically accompanied by chronic diarrhoea, widespread lesions of small vessels resulting in showers of petechiae (particularly during intercurrent infection), easy bruising and orthostatic acrocyanosis (6). Biochemical features of EE include reduced mitochondrial respiratory complex IV activity in skeletal muscle, persistent lactic acidaemia and markedly elevated urinary excretion of ethylmalonic acid (EMA) and methylsuccinic acid (7). Patients with milder expression of EE have been treated with supplementary L-carnitine, riboflavin, Q10, as well as other vitamin therapies, which may improve energy metabolism and reduce oxidative stress. The clinical hetero- geneity of EE was recently illustrated by monochorionic twins with the condition, where one had an episode of coma at age 3 and now has spastic quadraparesis and cannot speak. The other twin freely uses her upper extremities; pyramidal symptoms are limited to lower extremities, and she speaks two languages (8).

Any provisional diagnosis of EE should be verified by testing of the urine for the pathologic accumulation of EMA, methylsuccinic acid and/or C4-C6 acylglycines (isobutyryl-, isovaleryl-, 2-methylbutyryl- and hexanoylglycine). Serum levels of C4-C6 carnitines (butyryl-, isobutyryl-, isovaleryl- and hexanoylcarnitine) may also be elevated. Urine and blood test results obtained in EE may be similar to those noted in multiple acyl-CoA dehydrogenase deficiency (MADD) or short chain acyl-CoA dehydrogenase deficiency (SCADD), where elevated urinary EMA can exist with or without serum butyrylcarnitine elevation. Accordingly, MADD and SCADD are often included in the differential diagnosis of EE until definitive (genotype) testing can be performed $(9,10)$. At least in severe EE, the recurrent petechiae, orthostatic acrocyanosis and chronic diarrhoea seem to be specific for this condition. The massive urinary excretion of thiosulfate classically observed in EE has been experimentally replicated in a knockout Ethe1(-/-) murine model; the resultant sulfide tissue accumulation potently inhibits COX and short-chain fatty acid oxidation. The vasoactive and vasotoxic effects of this sulfide excess appear to explain the microangiopathy present in EE patients (5).

Genetic counselling should be offered when the diagnosis of $\mathrm{EE}$ is confirmed. In the present case, genetic sequencing identified the same novel mutation in both husband and wife, a highly unusual statistical event for rare mutations in non-consanguineous pairing. The couple was aware that the chance of any subsequent pregnancy being affected with EE was $25 \%$, and therefore sought reproductive endocrinology consultation to minimise this risk. While IVF with embryo biopsy and single gene pre-implantation genetic diagnosis was discussed, the couple decided to first utilise a less aggressive therapeutic approach of IUI using donor sperm. Although this treatment strategy did not incorporate native gametes from both partners, pre-IUI psychological counselling was provided, and the patients were determined to be satisfactory candidates for donor IUI. Parallel to this treatment, siblings of the carrier couple were offered similar testing to determine the carrier status of family members. While the patient did conceive from insemination with donor sperm, the baby was not healthy for reasons other than EE. It should be noted that, even if the couple had elected to undergo IVF with PGD, the anomaly encountered in the subsequent pregnancy would have escaped detection before embryo transfer.

In summary, this report presents a diagnostic evaluation and clinical management plan for fertility patients having a prior offspring with EE, and expands our knowledge of causative mutations for this disorder. While IVF incorporating pre-implantation genetic diagnosis could have been applied in this circumstance, our patient preferred to reserve deployment of IVF in favour of a more conservative approach. Additional clinical and laboratory research is needed to more fully characterise this rare condition, and definitive diagnosis remains a key objective before beginning any fertility treatment. 


\section{Acknowledgements}

The authors wish to express appreciation to the Institute of Clinical Research, Aarhus University and Aarhus University Hospital (Skejby, Denmark) for their support with the genotyping.

\section{References}

1. Tiranti V, D'Adamo P, Briem E, et al: Ethylmalonic encephalopathy is caused by mutations in ETHE1, a gene encoding a mitochondrial matrix protein. Am J Hum Genet 74: 239-252, 2004.

2. Mineri R, Rimoldi $M$, Burlina $A B$, et al: Identification of new mutations in the ETHE1 gene in a cohort of 14 patients presenting with ethylmalonic encephalopathy. J Med Genet 45: 473-478, 2008.

3. Quenby S, Kalumbi C, Bates M, Farquharson R and Vince G: Prednisolone reduces preconceptual endometrial natural killer cells in women with recurrent miscarriage. Fertil Steril 84: 980-984, 2005.
4. Abrahams VM: Mechanisms of antiphospholipid antibodyassociated pregnancy complications. Thromb Res 124: 521-525, 2009.

5. Tiranti V, Viscomi C, Hildebrandt T, et al: Loss of ETHE1, a mitochondrial dioxygenase, causes fatal sulfide toxicity in ethylmalonic encephalopathy. Nat Med 15: 200-205, 2009.

6. Tiranti V, Briem E, Lamantea E, et al: ETHE1 mutations are specific to ethylmalonic encephalopathy. J Med Genet 43: 340-346, 2006.

7. García-Silva MT, Ribes A, Campos Y, Garavaglia B and Arenas J: Syndrome of encephalopathy, petechiae and ethylmalonic aciduria. Pediatr Neurol 17: 165-170, 1997.

8. Pigeon N, Campeau PM, Cyr D, Lemieux B and Clarke JT: Clinical heterogeneity in ethylmalonic encephalopathy. J Child Neurol 24: 991-996, 2009.

9. Merinero B, Pérez-Cerdá C, Ruiz Sala P, et al: Persistent increase of plasma butyryl/isobutyrylcarnitine concentrations as marker of SCAD defect and ethylmalonic encephalopathy. J Inherit Metab Dis 29: 685, 2006.

10. Gregersen N, Andersen BS, Pedersen CB, Olsen RK, Corydon TJ and Bross P: Mitochondrial fatty acid oxidation defects remaining challenges. J Inherit Metab Dis 31: 643-657, 2008. 